\title{
Saccular aneurysms at middle basilar trunk fenestration
}

\author{
Luciano H.L. Foroni' ${ }^{1}$ Eberval G. Figueiredo ${ }^{1}$, Manoel J. Teixeira', \\ José G.M.P. Caldas², Alexandre Leszczynski², Fabiano R. Rivau²
}

Basilar artery (BA) fenestration is reported in $0.6 \%$ of angiograms ${ }^{1}$ and in about $5 \%$ of autopsy series ${ }^{2,3}$. It may occur anywhere along the BA but it is most frequent in the proximal trunk, close to the junction of the vertebral arteries ${ }^{2-5}$, and rarer in the middle and distal thirds. In these instances, they may represent therapeutic dilemmas. Surgical treatment of these aneurysms is difficult because of the complex geometry of the fenestration, the proximity to the lower cranial nerves, the presence of multiple small perforating arteries, and difficulties in obtaining adequate surgical exposure ${ }^{2-7}$. Endovascular treatment of such aneurysms has been described with success in most cases, with little or no neurological deficits ${ }^{1,8,9}$.

We report a rare case of two berry aneurysms originating in a fenestration of the BA and discuss its pathophysiology and therapeutic nuances.

\section{CASE REPORT}

A 55-year-old woman presented sudden headache. Neurological examination disclosed quadrantanopia at the left inferior visual field. Computed tomography (CT) demonstrated intraparenchymal hemorrhage at the right parietal lobe. Angiogram demonstrated aneurysms at the right posterior communicating artery (PComA) and anterior choroidal artery (AChA), and two small aneurysms at a BA fenestration (Fig 1A and 1B). The fenestration measured $5.5 \mathrm{~mm}$ in length and it was located at the transition between the middle and distal thirds (Fig 2A and 2B). Magnetic resonance imaging (MRI) demonstrated a small cavernous angioma, an angiographically occult lesion. The two anterior circulation aneurysms were clipped uneventfully. The two BA fenestration aneurysms were successfully treated with embolization, using Guglielmi detachable
Correspondence

Eberval G. Figueiredo

Rua Oscar Freire 1399 / 171

05409-010 São Paulo SP - Brazil

E-mail: ebgadelha@yahoo.com

Received 23 May 2009

Accepted 15 September 2009

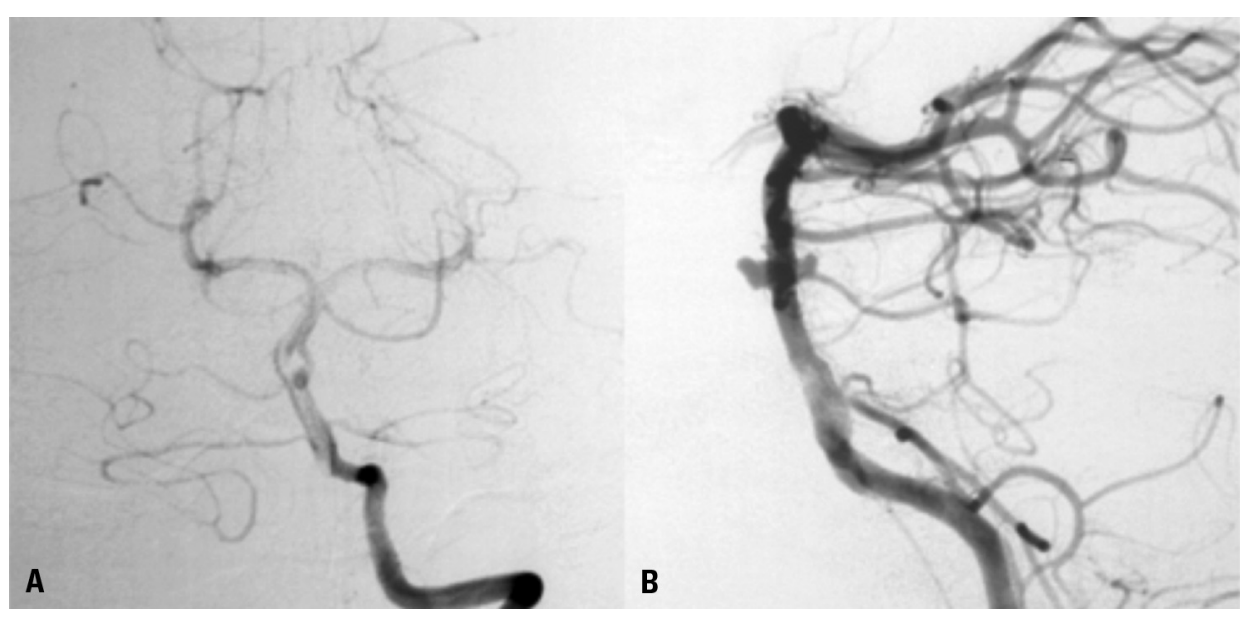

Fig 1. Preoperative angiogram depicts a mid-trunk BA fenestration giving rise to two small berry aneurysms. [A] Frontal view. [B] Lateral view.

\section{ANEURISMAS SACULARES NA FENESTRAÇÃO DA ARTÉRIA BASILAR}

1 Division of Neurosurgery, Hospital das Clínicas, University of São Paulo Medical School (USP) São Paulo, São Paulo SP, Brazil; ${ }^{2}$ Neuroradiological Intervention, Institute of Radiology, Hospital das Clínicas, University of São Paulo Medical School (USP) São Paulo, São Paulo SP, Brazil. 


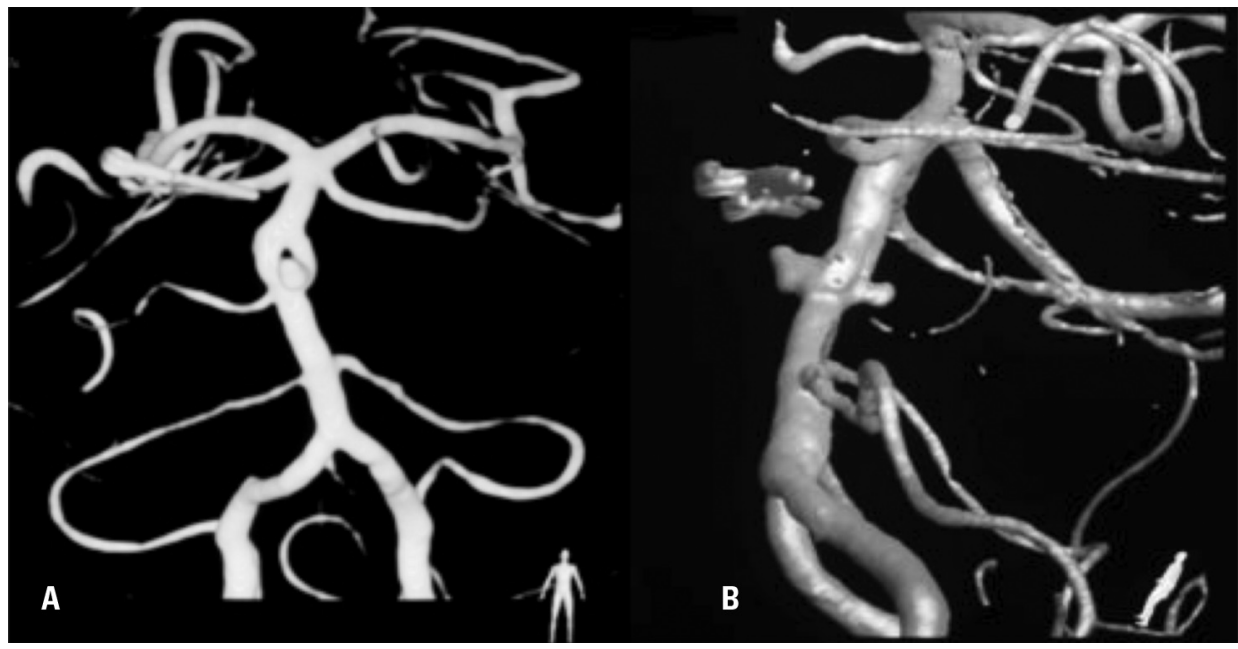

Fig 2. 3D angiogram of the BA demonstrates the relationship between the fenestration and the aneurysm. [A] Frontal view. [B] Lateral view.
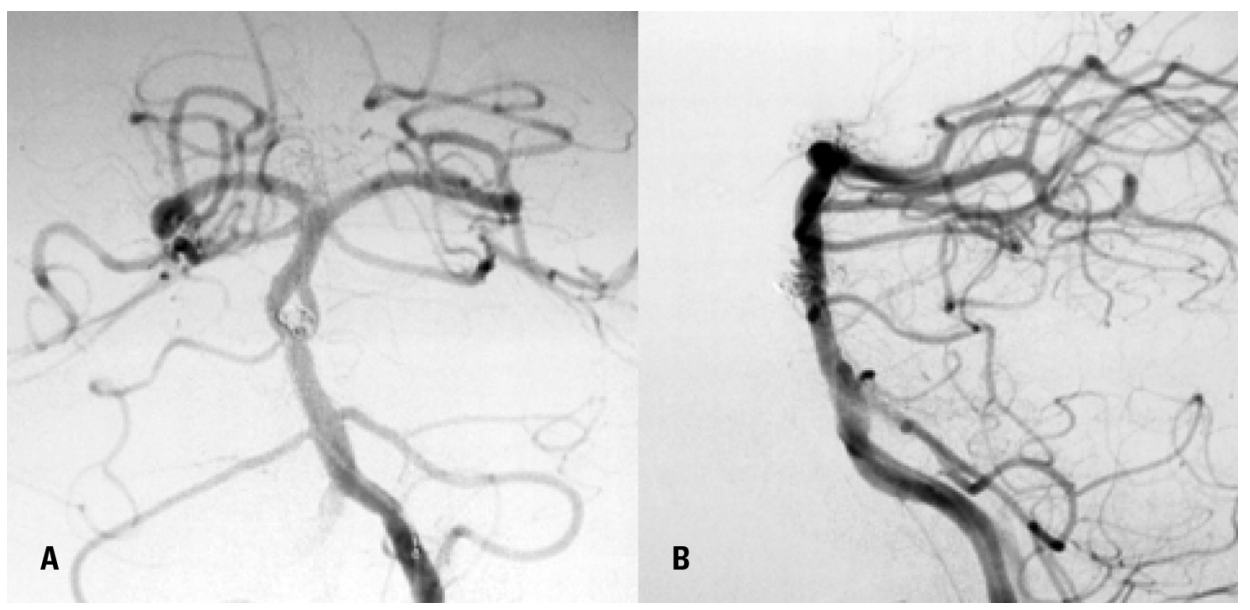

Fig 3. Post- embolization angiogram showing complete occlusion of the aneurysms. [A] Frontal view. [B] Lateral view.

coils, with preservation of the branches of fenestration (Fig $3 \mathrm{~A}$ and $3 \mathrm{~B}$ ) and no additional deficit. Patient has given her consent for publication of this report.

\section{DISCUSSION}

The BA is formed during the second to fourth stages of embryonic development, by the fusion of two primitive longitudinal neural arteries at the fifth fetal week ${ }^{3,4}$ ${ }^{6,9}$. In the early stages of fusion, these arteries are connected by several bridging areas and further fusion occurs to form the BA. If these bridges persist, they will result in fenestration or, if fusion fails to occur, duplication of the BA. Some cases of fenestration of the basilar artery may be caused by persistence of the cranial part of a primitive lateral vertebrobasilar anastomosis ${ }^{3,4-6,9}$. The angled junctions of the fenestrations have been shown to have defects of the tunica media at the proximal and distal ends ${ }^{3,4-6,9}$. These defects, in combination with the resulting alteration in flow characteristics and turbulence, are thought to predispose to the formation of aneurysms $5^{5,10}$, which occur particularly at the proximal end $\mathrm{d}^{4}$.

Fenestrations may be classified, according to their length, as small (0-3 mm), medium (3.1-5 $\mathrm{mm})$ and large $(>5.1 \mathrm{~mm})$. They have proved to be associated with defects on the wall at its proximal and distal margins ${ }^{4,7}$. It is thought that these defects and the change in flow characteristics, which occur at the site, predispose to the formation of aneurysms. Sanders et al. ${ }^{11}$ found only one aneurysm (7\% prevalence) at the site of fenestration in their series of 16 basilar artery fenestrations, identified in a retrospective review of 5190 angiograms. Tasker and Byrne ${ }^{12}$, in their series of 9 cases of fenestrations of the BA, in 6 
of them (66.7\% prevalence) the aneurysm arose at the site of fenestration.

To the best of our knowledge, this is the third time that two aneurysms at the same site of basilar artery fenestration have been reported ${ }^{5,12}$; or fourth, if we include the different sites of the same basilar artery fenestration?.

Cavernous angiomas may present (with) symptomatic hemorrhage in $20 \%$ of cases. They have a known association with other vascular malformations ${ }^{2}$, but there is no known pathophysiological association between cavernous angiomas and intracranial aneurysms. The management options for these lesions are expectant, surgical excision or stereotactic radio surgery (controversial).

Our patient did not present any other hemorrhage from the cavernous angioma or seizure during a 5-year follow-up. The anterior circulation and the BA fenestration aneurysms were successfully treated with surgical clipping and endovascular embolization, respectively.

In summary, we report a very rare case of two aneurysms originating at distal BA fenestration. The patient was managed with three distinct alternatives: expectant clinical follow-up of the cavernous angioma, surgical clipping of the anterior circulation aneurysms and endovascular embolization of the basilar artery fenestration aneurysms.

\section{REFERENCES}

1. Bavinzski G, Killer M, Gruber A, Reinprecht A, Gross CE, Richling B. Treatment of basilar artery bifurcation aneurysms by using Guglielmi Detachable coils: a 6-year experience. J Neurosurg 1999;90:843-852.

2. Awad IA, Robinson JR, Jr., Mohanty S, Estes ML. Mixed vascular malformations of the brain: clinical and pathogenetical considerations. Neurosurgery 1993;33:179-188.

3. Becker DH, Hamilton RD. Saccular aneurysm associated with fenestrated basilar artery: case report. Neurosurgery 1979;5:695-697.

4. Black SPW, Ansbacher LE. Saccular aneurysm associated with segmental duplication of the basilar artery. J Neurosurg 1984;61:1005-1008.

5. Campos J, Fox AJ, Vinuela F, et al. Saccular aneurysms in basilar artery fenestration. Am J Neuroradiol 1987;8:233-236.

6. De Caro R, Srafini MT, Galli S, Parenti A, Guidolin D, Munari PF. Anatomy of segmental duplication in the human basilar artery: possible site of aneurysm formation. Clin Pathol 1995;14:303-309.

7. Drake CG. The surgical treatment of aneurysms of the basilar artery. J Neurosurg 1968;29:436-446.

8. Eskridge JM, Song JK. Endovascular embolization of 150 basilar tip aneurysms with Guglielmi detachable coils: results of the Food and Drug Administration multicenter clinical trial. J Neurosurg 1998;89:81-86.

9. Saatci I, Cekirge HS, Karcaaltincaba M, et al. Endovascular treatment of kissing aneurysms at the fenestrated basilar artery: case report with literature review. Surg Neurol 2002;58:54-58.

10. Ferguson GG. Physical factors in initiation, growth and rupture of human intracranial saccular aneurysms. J Neurosurg 1972;37:666-667.

11. Sanders WP, Sorek PA, Mehta BA. Fenestration of intracranial arteries with special attention to associated aneurysms and other abnormalities. Am J Neuroradiol 1993;14:675-680.

12. Tasker AD, Byrne JV. Basilar artery fenestration in association with aneurysms of the posterior cerebral circulation. Neuroradiology 1997;39:185-189. 